BNL-45356

AD/RHIC-87

Conference Paper

\title{
The Transverse Damper System for RHIC
}

J. Xu, J. Claus, E. Raka, A. G. Ruggiero and T. J. Shea

May 1991

R H I C P R O J E C T

Brookhaven National Laboratory

Associated Universities, Inc.

Upton, NY 11973

Under Contract No. DE-AC02-76CH00016 with the

UNITED STATES DEPARTMENT OF ENERGY 


\title{
1991 IEEE Particle Accelerator Conference \\ San Francisco, CA \\ May 6-9, 1991
}

THE TRANSVERSE DAMPER SYSTEM FOR RHIC*

\author{
J. Xu, J. Claus, E. Raka, A.G. Ruggiero and T.J. Shea \\ Brookhaven National Laboratory \\ Upton, NY 11973, USA
}

\section{INTRODUCTION}

If the beam is injected with errors $x_{c}, x_{c}^{\prime}$ (or $y_{c}, y_{c}^{\prime}$ ) with respect to the closed orbit or disturbed by transverse instabilities, it will execute coherent oscillations and will be diluted in betatron phase space within a time interval of about $1 / \Delta \nu$ turns, even if it is properly matched to the focusing characteristics of the lattice, unless there is an effective damper system to prevent this. Here $\Delta \nu$ is the tune spread in the beam. Such a damper will not prevent dilution due to mismatches. Without such a damper the emittance of the beam will ultimately develop to a properly centered matched ellipse with an area $\epsilon$ in phase space that is larger than that of the injected one $\epsilon_{0}$ which is also matched but off-centered by $x_{c}$ and $x_{c}^{\prime}$.

Let the equations of the centered ellipse and the injected off-centered but matched ellipse be[1]

$$
\epsilon=\gamma x^{2}+2 \alpha x x^{\prime}+\beta x^{\prime 2}
$$

and

$$
\epsilon_{0}=\gamma\left(x-x_{c}\right)^{2}+2 \alpha\left(x-x_{c}\right)\left(x^{\prime}-x_{c}^{\prime}\right)+\beta\left(x^{\prime}-x_{c}^{\prime}\right)^{2}
$$

respectively, where $\alpha, \beta, \gamma$ are the Twiss parameters at the injection point.

The dilution factor $\epsilon / \epsilon_{0}$ can be expressed as follows:

$$
\frac{\epsilon}{\epsilon_{0}}=\left[1+\sqrt{\frac{\epsilon_{c}}{\epsilon_{0}}}\right]^{2}
$$

where $\epsilon_{c}=\gamma x_{c}^{2}+2 \alpha x_{c} x_{c}^{\prime}+\beta x_{c}^{\prime 2}$. We have similar relations for the $y$-direction.

If we require an emittance growth less than $20 \%$ in both directions, the maximum allowable values are $x_{c}$ $=0.25 \mathrm{~mm}, x_{c}^{\prime}=0.03 \mathrm{mrad}, y_{c}=0.6 \mathrm{~mm}$ and $y_{c}^{\prime}=$ 0.012 mrad. These tolerances may be difficult to meet and a feedback system to damp the coherent oscillations induced by the injection errors should be considered for RHIC.

The damper system consists of a beam position monitor, signal processing electronics, an amplifier station and a kicker device. The beam position signal is amplified and transported to another location where it is applied across the kicker device for the correction. Each kicker is made of a pair of striplines of length $\ell$. If $\pm V_{k}$ is the voltage applied to each plate then the effect of the kicker on the ion motion can be expressed as follows:

\footnotetext{
* Work performed under the auspices of the U.S. Department of Energy.
}

$$
A m_{0} \gamma \ddot{x}=\frac{2 Z e V_{k}}{d_{k}} \eta \alpha
$$

where $Z$ is the charge state, $A$ the mass number, $m_{0}$ the specific mass at the rest of the ion, $d_{k}$ the plate separation and $\gamma$ the relativistic energy factor. The factor $\eta$ describes the interaction between the kicker and the beam. There are contributions from both electric and magnetic fields. If the kicker plates are terminated at the upstream end with the characteristic impedance, the two contributions add and $\eta \simeq 2$. The average electric field in the kicker is approximately $2 V_{k} / d_{k}$. The factor $\alpha$ measures the enhancement or depression of the electric and magnetic fields at the center of the vacuum chamber due to the geometrical configuration of the kicker design.

The kick angle received by an ion going through the system of kickers is given by

$$
\theta=\frac{2 Z e n_{k} \ell V_{k}}{A E_{0} \gamma \beta^{2} d_{k}} \tau \eta \alpha
$$

where $n_{k}$ is number of kickers each of length $\ell, E_{0}=m_{0} c^{2}$, $\beta$ is the relativistic velocity and $\tau$ is a transit-time factor which in the frequency domain $\omega$ is expressed as

$$
\tau=\frac{\sin \frac{2 \omega \ell}{c}}{\frac{2 \omega \ell}{c}}
$$

In the following we have assumed a square voltage pulse applied to the damper kicker, with a duration long enough so that $\tau=1$.

For a given damper system we will consider two modes of operation: (i) the kicker voltage is proportional to the beam position signal and (ii) the kicker voltage is adjusted to a constant value having a sign which depends on the sign of the signal detected from the beam position.

\section{PROPORTIONAL MODE[1]}

In the proportional mode the correction effect is proportional to the beam center displacement. As the damping proceeds, the signal and therefore the correction are reduced. The power required at any time is then proportional to the square of the beam displacement, and is the highest at the beginning.

Let $X_{p, n} \equiv\left(x_{p, n}, x_{p, n}^{\prime}\right)$ be the vector representing the coherent oscillation at the pick-up location during the n-th crossing. The kicker voltage $V_{k n}$ during n-th crossing of the kicker is proportional to $x_{p n}$,

$$
V_{k n}=-G x_{p, n}
$$


then the vector during the $(n+1)$-th crossing is

$$
X_{p, n+1}=M_{k p} M_{k} M_{p k} X_{p, n}
$$

where $M_{p k}$ and $M_{k p}$ are the $2 \times 2$ transfer matrices respectively from pick-up to kicker and from kicker to pick-up and $M_{k}$ is the matrix representing the effect of the kicker. We have

$$
\begin{aligned}
M_{k} & =\left(\begin{array}{ll}
1 & 0 \\
0 & 1
\end{array}\right)-k_{0}\left(\begin{array}{ll}
0 & 0 \\
1 & 0
\end{array}\right) M_{p k}^{-1} \\
& =I-k_{0} R M_{p k}^{-1}
\end{aligned}
$$

where

$$
k_{0}=\frac{2 Z e n_{k} \ell G}{A E_{0} \gamma \beta^{2} d_{k}} \eta \alpha=\frac{\theta_{n}}{x_{p, n}}
$$

is a damper parameter that can be obtained by combining Eqs. $(5,7)$.

We have

$$
\begin{aligned}
M & =M_{k p} M_{k} M_{p k} \\
& =M_{0}-k_{0} M_{k p} R
\end{aligned}
$$

where $M_{0}=M_{k p} M_{p k}$ is the unperturbed transfer matrix at the pick-up location.

Let

$$
M_{0}=\left(\begin{array}{ll}
m_{11} & m_{12} \\
m_{21} & m_{22}
\end{array}\right)
$$

and

$$
M_{k p} R=\left(\begin{array}{ll}
a & 0 \\
b & 0
\end{array}\right)
$$

with

$$
\begin{aligned}
& a=\sqrt{\beta_{k} \beta_{p}} \sin \psi_{k p} \\
& b=\sqrt{\frac{\beta_{k}}{\beta_{p}}}\left(\cos \psi_{k_{p}}-\alpha_{p} \sin \psi_{k p}\right)
\end{aligned}
$$

where $\psi_{k p}$ is the phase advance from kicker to beam monitor. The damping rate is given by the eigenvalues of the total transfer matrix $M$, which are the solutions of the quadratic equation

$$
\lambda^{2}-\lambda\left(2 \cos \mu_{0}-k_{0} a\right)+\operatorname{Det} M=0
$$

where $\mu_{0}$ is the unperturbed phase advance per revolution. If, finally, we let

$$
k=k_{0} \sqrt{\beta_{p} \beta_{k}}
$$

then

$$
\lambda=\cos \mu_{0}-\frac{k}{2} \sin \psi_{k p} \pm i \sqrt{\left(\sin \mu_{0}+\frac{k}{2} \cos \psi_{k p}\right)^{2}-\left(\frac{k}{2}\right)^{2}} .
$$

The case of practical interest is when the phase advance $\psi_{p k}$ from monitor to the kicker is adjusted close to an odd number of $\pi / 2$ radians and the gain $k$ is small. In this case, apart from a phase factor of no consequence, the damping rate of the coherent oscillations is given by $\frac{1}{2} k \sin \psi_{p k}$ per turn or $\frac{1}{2} \frac{\theta}{x_{p}} \sqrt{\beta_{p} \beta_{k}} \sin \psi_{p k}$ per turn. Since $\sin \psi_{p k} \sim \pm 1$ the damping rate is simply given by

$$
D_{p r}=\frac{1}{2} \frac{\theta}{x_{p}} \sqrt{\beta_{p} \beta_{k}} \text { per turn }
$$

\section{CONSTANT VOLTAGE MODE[2]}

In the constant voltage mode the beam position signal is used only to trigger the correction by applying a constant kicker voltage. It also controls the sign of the voltage according to the actual sign measured of the instantaneous beam displacement. This method still provides effective damping but with a considerably reduced peak power requirement.

The Courant-Snyder invariant of the coherent oscillation at the n-th turn, at the kicker location, is given by

$$
A_{n}^{2}=\gamma_{k} x_{k, n}^{2}+2 \alpha_{k} x_{k, n} x_{k, n}^{\prime}+\beta_{k} x_{k, n}^{2}
$$

where $x_{k, n}$ and $x_{k, n}^{\prime}$ are the $x$ and $x^{\prime}$ values during $n$-th crossing of the kicker. Let $\theta_{n}$ be the kick applied, then assuming the position is unchanged, we have a change in $A_{n}$ given by

$$
\Delta A_{n}^{2}=2\left(\alpha_{k} x_{k, n}+\beta_{k} x_{k, n}^{\prime}\right) \theta_{n}+\beta_{k} \theta_{n}^{2}
$$

If the correction is small we can neglect the quadratic term. Using explicitly equations for $x_{k, n}$ and $x_{k, n}^{\prime}$, we obtain

$$
\Delta A_{n}=\beta_{k}^{\frac{1}{2}} \theta_{n} \cos \psi_{k, n}
$$

where $\psi_{k . n}$ is the phase of the coherent oscillation during $n$-th crossing of the kicker which varies from turn to turn.

For the correction kicker, we take

$$
\theta_{n}=-\theta_{0} \frac{\left|\cos \psi_{k, n}\right|}{\cos \psi_{k, n}}
$$

where $\theta_{0}$ is a constant. We have finally

$$
\Delta A_{n}=-\beta_{k}^{\frac{1}{2}} \theta_{0}\left|\cos \psi_{k, n}\right|
$$

which gives the variation of the Courant-Snyder invariant of the coherent oscillation during $n$-th crossing of the kicker. Then, $A_{N}$ at the $\mathrm{n}$-th turn can be expressed as

$$
A_{N}=A_{0}-\sum_{n=1}^{N} \beta_{k}^{\frac{1}{2}} \theta_{0}\left|\cos \psi_{k, n}\right|
$$

We can replace $\sum_{n=1}^{N}\left|\cos \psi_{k, n}\right|$ with $N \overline{|\cos \psi|}$ and obtain the following expression:

$$
A_{N}=A_{0}-N \frac{2}{\pi} \beta_{k}^{\frac{1}{2}} \theta_{0} .
$$

The average fractional reduction of the coherent oscillation amplitude per turn when it reduces from $x_{0}$ to $x_{0} e^{-1}$ is

$$
D_{c}=\frac{2}{\pi} \frac{\sqrt{\beta_{p} \beta_{k}} \theta_{0}}{x_{0}\left(1-e^{-1}\right)} \simeq \sqrt{\beta_{p} \beta_{k}} \frac{\theta_{0}}{x_{0}}
$$


Comparing Eqs. (18) and (26), one finds that the effective damping rate with the constant voltage mode is about twice that obtained with the proportional mode. The constant voltage mode is proposed to allow a reduction by a factor of 4 of the total power requirement. When the residual coherent oscillation amplitude becomes small enough one may switch to the proportional mode.

\section{- IV. RHIC PARAMETERS}

As determined in ref.[1], the most demanding requirements are set by injection of the beam of gold ion $(\mathrm{Z}=79, \mathrm{~A}=197)$ at $10.5 \mathrm{GeV} / \mathrm{u}$. An initial beam center displacement translated at the location of the pickup, as large as $2 \mathrm{~mm}$ can be expected from injection errors. It is required that damping take place in about one hundred turns $(\Delta \nu \simeq 0.01$ ), which is fast enough to avoid emittance dilution due to the beam tune spread. We take $\sqrt{\beta_{p} \beta_{k}} \sin \psi_{p k}=50 \mathrm{~m}$, from Eq. (23) one gets $\theta_{0}=0.4 \mu \mathrm{rad}$, which can be maintained with

$$
n_{k} \ell V_{k}=200 \mathrm{~V} \cdot \mathrm{m}
$$

here we take $\eta=2, \alpha=1, \tau=1$ in Eq. (5).

The total power required is

$$
P_{k}=2 n_{k} \frac{V_{k}^{2}}{R_{k}}
$$

where $R_{k}$ is the termination impedance which we take here to be $50 \Omega$. A reasonable solution is one set of kicker $\left(n_{k}=1\right) \ell=2$ meter long. On each plate a voltage 100 volts is applied which requires a total power of 400 watts.

The rise and fall times of the damper system should be short enough to allow individual damping of coherent motion of up to $3 \times 57$ bunches.

\section{REFERENCES}

[1] J. Xu, J. Claus and A.G. Ruggiero, BNL AD/RHIC74, July 1990.

[2] J. Xu and A.G. Ruggiero, BNL AD/RHIC-76, August 1990. 